\title{
Nonlinear friction of a damped dimer sliding on a periodic substrate
}

\author{
S. Gonçalves, ${ }^{1,2, *}$ V. M. Kenkre, ${ }^{1, \dagger}$ and A. R. Bishop ${ }^{3, \dagger}$ \\ ${ }^{1}$ Consortium of the Americas for Interdisciplinary Science and Department of Physics and Astronomy, University of New Mexico, \\ Albuquerque, New Mexico 87131, USA \\ ${ }^{2}$ Instituto de Física, Universidade Federal do Rio Grande do Sul, Caixa Postal 15051, 90501-970 Porto Alegre RS, Brazil \\ ${ }^{3}$ Theoretical Division and Center for Nonlinear Studies, Los Alamos National Laboratory, Los Alamos, New Mexico 87545, USA
}

(Received 23 July 2004; published 12 November 2004)

\begin{abstract}
The nonlinear sliding friction of a dimer over a substrate is studied within a one-dimensional model, consisting of a vibrating dimer (two masses connected by a spring), internally damped, sliding over a sinusoidal potential. Molecular dynamics simulations show that the friction force has an approximate $v^{-3}$ dependence if the velocity is sufficiently large, and that there is a striking periodic variation of the proportionality coefficient with the ratio of the length of the dimer to the substrate wavelength. The nonlinear velocity dependence was predicted earlier for a Langevin model of an adsorbed layer in the presence of strong external force. We study it here in detail in the transient regime and without external force. We obtain the dependence on key parameters (internal dissipation, dimer mass, substrate corrugation, and length ratio), and examine the validity of the friction law. A semianalytical expression is suggested which confirms the numerical observations in the high-velocity regime.
\end{abstract}

DOI: 10.1103/PhysRevB.70.195415

PACS number(s): 81.40.Pq, 46.55.+d

\section{INTRODUCTION}

In the last two decades much progress has been made in the fundamental understanding of the origin of friction. ${ }^{1}$ The significance of this progress is particularly clear if we compare it with what had been done in the centuries after the friction laws were stated by Amonton and Coulomb, and elaborated upon by Leonardo da Vinci. ${ }^{2}$ Microscopic experiments on friction, ${ }^{3}$ and subsequent theory and simulation were started only in the last 15 years. ${ }^{4-7}$ Of the work that remains to be done to achieve a coherent picture of this complex phenomenon, an important aspect concerns nonlinear sliding friction. In his seminal paper, ${ }^{8}$ Persson presented numerical simulations in the nonlinear regime of an adsorbate sliding over a periodic potential. He used a Langevin model of interacting adlayer particles over a three-dimensional potential periodic in the plane. Such a model is reasonable for experiments of the kind performed by means of the quartz microbalance. ${ }^{3}$ In addition to analyzing the model numerically, Persson found analytically the dependence of the adlayer steady state velocity on the applied force and showed that the nonlinear sliding friction becomes linear at high values of the external force. Recently, Fusco and Fasolino ${ }^{9}$ reported numerical findings of the sliding friction of a dimer (two atoms attached by a spring) when the dimer moves over a one-dimensional periodic potential and is additionally subjected to external dissipation. They considered different values of the dimer-substrate commensuration ratio.

Ten years separate the original finding of Persson and the recent study of Fusco and Fasolino. Yet, there is no "experimental" confirmation of the nonlinear friction law, nor a study of its validity, or its dependence on the substrate topology. Several questions may be raised: What happens in the high velocity regime in the absence of the external force? What is the dependence of the nonlinear friction on the shape of the substrate? Is the nonlinear friction of a dimer different from that observed in an extended adsorbate? In order to answer the first two of these questions, we present here results for a sliding dimer over a periodic substrate potential. To focus on the nonlinear part of the friction, and at the same time to improve the signal-to-noise ratio, we perform simulations at zero temperature and without external dissipation: friction acts, in our model, only on the internal motion. We focus on the time behavior of this dimer in the absence of external forces. We compare our results with the prediction of Persson for the adsorbate with external force, and look for similarities and differences. We also suggest an analytical explanation supporting our results.

\section{MODEL}

A single particle moving in a periodic potential provided by the substrate would not exhibit friction as its energy would be repeatedly transformed from potential to kinetic, with no overall depletion. Some studies have addressed the effective friction of such a particle in the presence of noise. Such noise can be externally imposed ${ }^{10,11}$ or produced effectively by the motion of a harmonic chain of atoms (rather than a fixed potential) as representing the substrate. ${ }^{12} \mathrm{We}$ consider the system in the absence of noise. In the sliding object, let a second particle be attached to the first. A part of its sliding energy can be converted into internal vibrational energy. It is trivial to see that the equations

$$
\begin{aligned}
& m \ddot{x}_{1}=+k\left(x_{2}-x_{1}-a\right)+\frac{2 \pi u_{0}}{b} \sin \left(\frac{2 \pi x_{1}}{b}\right), \\
& m \ddot{x}_{2}=-k\left(x_{2}-x_{1}-a\right)+\frac{2 \pi u_{0}}{b} \sin \left(\frac{2 \pi x_{2}}{b}\right),
\end{aligned}
$$

where $x_{1,2}$ are the coordinates of the two particles of equal mass $m$, and $k, a, b, u_{0}$ are, respectively, the spring constant, equilibrium length of the dimer, wavelength of the substrate 


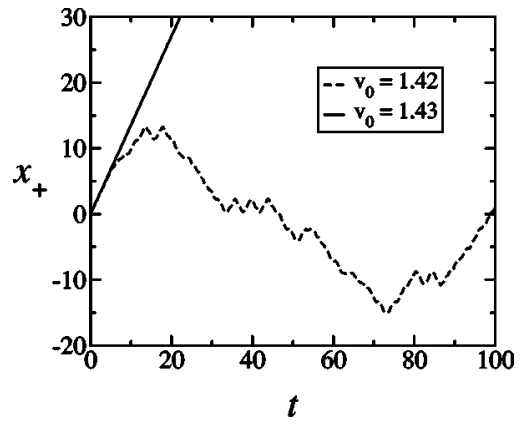

FIG. 1. Evolution of the center of mass coordinate $x_{+}$in the absence of dissipation. Plotted is $x_{+}$vs time $t$ from Eqs. (1) for two different initial velocities, below and above the upper chaos threshold, respectively; $x_{+}$is expressed in units of $b, t$ is in units of $b \sqrt{m / u_{0}}$, and $v_{0}$ is in units $\sqrt{u_{0} / m}$. In the present case $a=1.4 b$ and $k=2 u_{0} / b^{2}$.

potential and half the amplitude of the potential, which lead to coupled equations for the center of mass coordinate $x_{+}$and the internal coordinate $x_{-}$. The latter are defined as half the sum and difference of $x_{1,2}$, respectively. It is the presence (and additionally, the nonlinearity) of the substrate potential that is responsible for the coupling between the otherwise independent coordinates $x_{+}$and $x_{-}$. Analytic solutions of the coupled equations do not appear possible. The dynamics can be very complex depending on the initial energy, and numerical procedures become necessary.

The "normal" behavior of the dimer includes oscillations within potential barriers for low initial energy, and ballistic motion for high initial energy but with a velocity periodically modulated by the substrate potential. As the initial energy is increased, this oscillation becomes smaller compared to the dc component. For an intermediate narrow range of initial energies, the evolution is unpredictable and exhibits chaos. As an illustration of some of this behavior, we show in Fig. 1 the center of mass evolution for two different initial velocities, below and above the upper chaos threshold, respectively. The behavior in the intermediate region shows three characteristics as a result of the interchange of energy between translational and vibrational modes: forward movement, backward movement, and temporarily no movement represented by a plateau in the plot (around $t \approx 40$ in Fig. 1). However, this last is an unstable situation, as the system is conservative: the internal energy can be reconverted into translational energy allowing the dimer to slide again. This is repeated forever in the absence of dissipation. Therefore, the undamped dimer in a periodic potential is either trapped, or executes a ballistic movement, or is in diffusive chaotic motion in the intermediate energy region. Such nonlinear dynamics of a dimer on a periodic substrate, without dissipation, was thoroughly addressed recently by Fusco, Fasolino, and Janssen. ${ }^{13}$

To make the system more realistic, in the present paper we assume that the internal mode is subjected to damping at rate $\gamma$. This results in the following equations for the coordinates $x_{+,-}$:

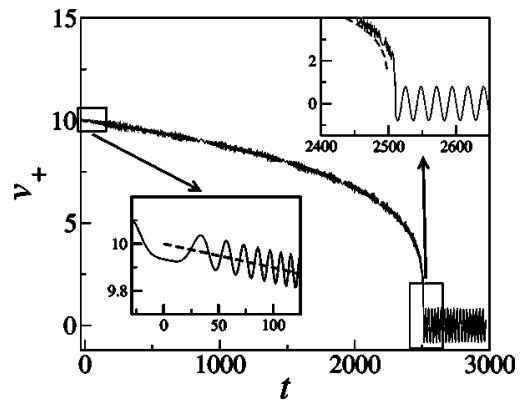

FIG. 2. Evolution of the center of mass velocity $v_{+}(t)$ in the presence of damping applied to the internal coordinate. Plotted is $v_{+}(t)$ in units of $\sqrt{u_{0} / m}$ vs time $t$ in units of $b \sqrt{m / u_{0}}$. Parameters here are $a / b=0.25, k b^{2} / u_{0}=1$, and $\gamma b \sqrt{m / u_{0}}=4$. Two insets show the detail of oscillations at times much smaller (left lower inset) and larger (right upper inset) than the stopping time. Solid lines in the inset show the numerical results while the dashed lines represent our proposed friction law.

$$
\begin{gathered}
\ddot{x}_{+}=\frac{2 \pi u_{0}}{m b} \sin \left(\frac{2 \pi x_{+}}{b}\right) \cos \left(\frac{2 \pi x_{-}}{b}\right), \\
\ddot{x}_{-}=-\frac{k}{m}\left(2 x_{-}-a\right)+\frac{2 \pi u_{0}}{m b} \sin \left(\frac{2 \pi x_{-}}{b}\right) \cos \left(\frac{2 \pi x_{+}}{b}\right) \\
-\gamma \dot{x}_{-}
\end{gathered}
$$

While dissipation removes chaos from the system, the coupling between coordinates makes it impossible to produce an analytical solution of Eq. (2). To proceed numerically, we use the algorithm of Verlet modified to allow for velocity dependent forces. Thus, if $x$ is a generic coordinate, $\Delta t$ is a time increment, and $\zeta(t)$ is the acceleration corresponding to the forces not depending on velocities, we use

$$
x(t+\Delta t)=\frac{2 x(t)-(1-\gamma \Delta t / 2) x(t-\Delta t)+\zeta(t) \Delta t^{2}}{1+\gamma \Delta t / 2} .
$$

\section{NUMERICAL RESULTS AND PHENOMENOLOGICAL LAW}

Typical evolution of the center of mass velocity from simulations of our model is in Fig. 2, and exhibits two distinct features: The rate of decrease of the center of mass velocity rises sharply as time evolves; and the velocity appears to go to zero (except for the persistent small-amplitude oscillations) in finite time. Thus, we see that effective friction emerges from the conversion of the translational to internal motion (which has an intrinsic sink), and that this friction is nonlinear. It has an inverse dependence on velocity, being stronger at lower velocities. Indeed, it appears to obey approximately the friction law $d v / d t=-\eta v^{-\alpha}$, with solution

$$
\frac{v(t)}{v_{0}}=\left[1-\frac{(\alpha+1) \eta t}{v_{0}^{\alpha+1}}\right]^{\frac{1}{\alpha+1}},
$$

where $v_{0}$ is the initial velocity. Equation (4) predicts a stopping time $t_{s}$ given by 


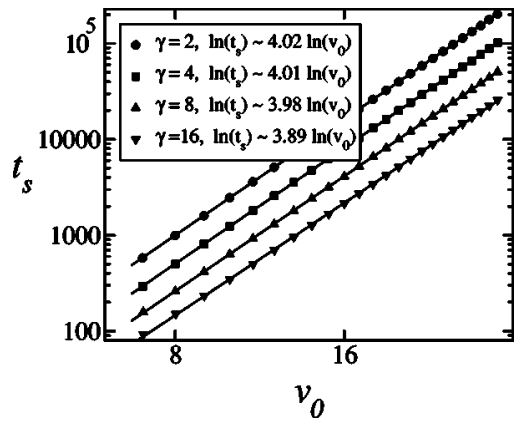

FIG. 3. Evidence for our proposed power law $(\alpha=3)$. Shown is the relation between the apparent stopping time $t_{s}$ and the initial velocity $v_{0}$, for $a / b=2$ and different sets of $\gamma$, and $k$. Velocity and the stopping time are given in units of $\sqrt{u_{0} / m}$ and $b \sqrt{m / u_{0}}$, respectively; $\gamma$ is in units of $\sqrt{\left(u_{0} / m\right)} / b$ and $k$ is in units of $u_{0} / b^{2}$. The lines are a linear regression of the log-log relation given consistently by an exponent $\approx 4$, thus $\alpha \approx 3$.

$$
t_{s}=\frac{v_{0}^{\alpha+1}}{(\alpha+1) \eta} .
$$

We plot in Fig. 3 the velocity dependence of the numerically obtained apparent stopping time and interpret it in terms of Eq. (5). The plot provides clear evidence that the exponent in expression (5) is $\alpha \approx 3$, with less than $2 \%$ error.

How does the coefficient $\eta$ of this sliding friction depend on the topology and dynamics of the system? To answer this question, we vary the parameters $a, b, u_{0}, k, m$, and $\gamma$, and the initial velocity $v_{0}$ over wide regions. In fact it is only necessary to do this for the relevant dimensionless quantities $a / b, k b^{2} / u_{0}$, and $\gamma b \sqrt{m / u_{0}}$, and the initial dimensionless velocity $v_{0} \sqrt{m / u_{0}}$. The striking results are in Fig. 4. In addition to confirming that the coefficient of proportionality $\eta$ is directly proportional to $\gamma,{ }^{8}$ and also proportional to $\left(u_{0} / m\right)^{2}$, but independent of $k$, we find an important result. The coefficient $\eta$ has a periodic dependence on $a / b$ fitted excellently by a simple sinusoid. All these results may be combined into the following phenomenological friction law we propose:

$$
\frac{d v}{d t}=-\frac{\gamma}{2}\left(\frac{u_{0}}{m}\right)^{2} \sin ^{2}\left(\frac{\pi a}{b}\right) \frac{1}{v^{3}} .
$$

Agreement of the predictions of our law with numerical simulations is remarkably good provided the initial velocity

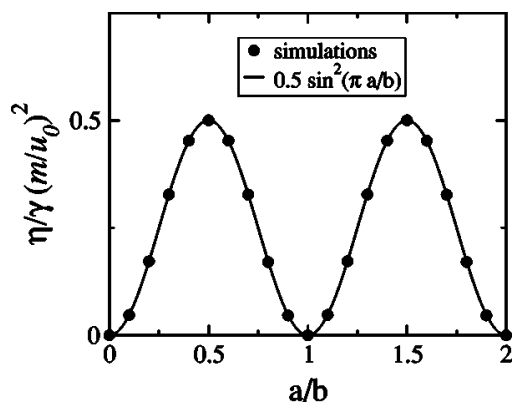

FIG. 4. Periodic dependence of the friction coefficient $\eta$ on the ratio of the dimer length to substrate wavelength. Plotted is $\eta$ in units of $\gamma\left(u_{0} / m\right)^{2}$ vs $a / b$.

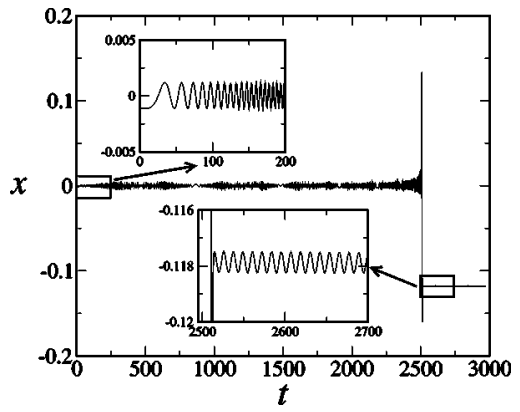

FIG. 5. Internal coordinate $x=x_{-}-a / 2$ vs time $t$. Note the relatively small amplitude of the oscillation supporting the $x \ll a / 2$ assumption; $x$ is in units of $b$, time is in units of $b \sqrt{m / u_{0}}$, and parameters are the same as in Fig. 2.

is large enough and the times are not too close to the stopping time. We will elaborate on its limitations below.

\section{ANALYTICAL CALCULATION}

The internal coordinate $x_{-}$has the natural frequency $\omega_{0}$ $=\sqrt{2 k / m}$. When scaled and translated through $\xi=\left(x_{-} / a / 2\right.$ -1 ), the internal coordinate obeys [see Eq. (2)] the damped driven oscillator equation

$$
\begin{aligned}
\frac{d^{2} \xi}{d t^{2}}+\gamma \frac{d \xi}{d t}+\omega_{0}^{2} \xi= & \left(\frac{4 \pi u_{0}}{a m b}\right) \sin \left[\left(\frac{\pi a}{b}\right)(1+\xi)\right] \\
& \times \cos \left(\frac{2 \pi x_{+}(t)}{b}\right) .
\end{aligned}
$$

The complexity of this equation relative to that for a harmonic oscillator driven linearly thorough a sinusoidally varying forcing term arises from two factors that appear on the right hand side: the $\xi$ dependence of the sin term and the generally nonsinusoidal $t$ dependence of the cos term. In Fig. 5 we have shown the behavior of the internal coordinate in a typical case. We see there that the internal coordinate has a small amplitude of oscillation throughout the evolution. If we restrict our attention only to such a case, $\xi$ may be neglected with respect to 1 in the sin term above. Generally, we may write, with $x_{+}(0)=0$ without loss of generality

$$
\cos \left(\frac{2 \pi x_{+}(t)}{b}\right)=\cos \left(\frac{2 \pi}{b} \int_{0}^{t} v(s) d s\right)=\sum_{i} B_{i} \cos \left(\omega_{i} t\right),
$$

where the complex $t$ dependence of the center of mass velocity has been resolved into Fourier components of frequencies $\omega_{i}$ and coefficients $B_{i}$. With the notation $A_{i}=(2 / a)$ $\times\left(2 \pi u_{0} / m b\right) \sin (\pi a / b) B_{i}$, we therefore study the linear damped oscillator

$$
\frac{d^{2} \xi}{d t^{2}}+\gamma \frac{d \xi}{d t}+\omega_{0}^{2} \xi=\sum_{i} A_{i} \cos \left(\omega_{i} t\right)
$$

whose behavior is known from textbooks. The coordinate $\xi$ may be decomposed into components $\xi_{i}$ each of which satisfies, after transients have died down 


$$
\xi_{i}(t)=\frac{A_{i}}{\sqrt{\left(\omega_{0}^{2}-\omega_{i}^{2}\right)^{2}+\omega_{i}^{2} \gamma^{2}}} \cos \left(\omega_{i} t-\delta_{i}\right),
$$

where the lag factor $\delta_{i}$ equals $\tan \delta_{i}=\omega_{i} \gamma /\left(\omega_{0}^{2}-\omega_{i}^{2}\right)$ for each Fourier component. Dissipation of energy from the center of mass motion into the internal coordinates occurs at the rate $R$ given by $2 m \gamma\left(d x_{-} / d t\right)^{2}=2 m \gamma(a / 2)^{2} \Sigma_{i}\left(d \xi_{i} / d t\right)^{2}$ whose average, since the square of the sinusoidal term contributes $1 / 2$, is

$$
\begin{aligned}
R & =m \gamma\left(\frac{a}{2}\right)^{2} \sum_{i} \frac{A_{i}^{2} \omega_{i}^{2}}{\left(\omega_{0}^{2}-\omega_{i}^{2}\right)^{2}+\omega_{i}^{2} \gamma^{2}} \\
& =m \gamma\left(\frac{2 \pi u_{0}}{m b}\right)^{2} \sin ^{2}\left(\frac{\pi a}{b}\right) \sum_{i} \frac{B_{i}^{2} \omega_{i}^{2}}{\left(\omega_{0}^{2}-\omega_{i}^{2}\right)^{2}+\omega_{i}^{2} \gamma^{2}} .
\end{aligned}
$$

We will now restrict our analysis to situations and time domains in which we can replace the $i$ sum by a single term involving an average frequency $\omega_{a}$, which may be weakly dependent on time. We then have

$$
\cos \left(\frac{2 \pi}{b} \int_{0}^{t} v(s) d s\right) \approx \cos \left(\omega_{a} t\right),
$$

$\omega_{a}$ being related to the center of mass velocity $v(t)$ through $\omega_{a}=2 \pi v(t) / b$ (the "washboard" frequency). Consider now the case that the washboard frequency $\omega_{a}$ is much larger than the natural frequency $\omega_{0}$ and the damping rate $\gamma$. We can then write

$$
R=m \gamma\left(\frac{2 \pi u_{0}}{m b}\right)^{2} \sin ^{2}\left(\frac{\pi a}{b}\right)\left(\frac{1}{\omega_{a}}\right)^{2} .
$$

Equating the rate at which the internal mode gains energy to that at which the center of mass loses it, viz., $-2 m v(d v / d t)$, we get the equation for the center of mass velocity

$$
\frac{d v}{d t}=-\frac{\gamma}{2}\left(\frac{u_{0}}{m}\right)^{2} \sin ^{2}\left(\frac{\pi a}{b}\right) \frac{1}{v^{3}} .
$$

This is precisely the result (6) we proposed above on the basis of an empirical inspection of our numerical results.

The analytical argument we have presented is valid under three assumptions. The first is that the amplitude of the internal dimer coordinate always remains small relative to the equilibrium value of the length of the dimer $(\xi \ll 1)$. The second is that the characteristic rate of variation of the center of mass velocity, viz., $(1 / v)(d v / d t)$, is smaller than both the natural frequency of the internal coordinate, $\omega_{0}$, and the dissipation rate, $\gamma$. The third assumption is that the washboard frequency $\omega_{a}$ which is proportional to the center of mass velocity $v$ is itself larger than the other characteristic frequencies, viz., $\omega_{0}$ and $\gamma$. Of these three assumptions, the last is easiest to realize practically or understand physically. It is valid provided we take the initial velocity to be sufficiently large and the time considered not too close to the stopping time. The specific values of the initial velocity required for this validity are determined by the specific given values of the system parameters $\omega_{0}$ and $\gamma$. In order to study the applicability of the assumptions, we present Fig. 6, where we compare the behavior of the dimer velocity according to ex-

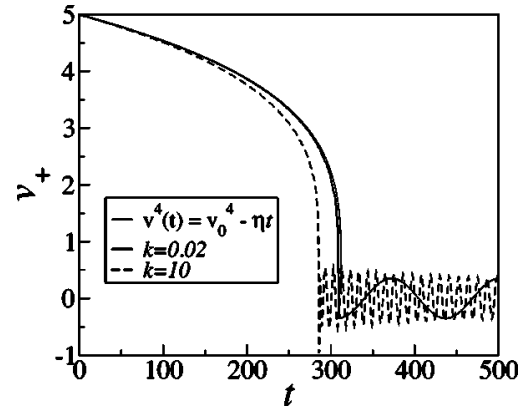

FIG. 6. Comparison between the analytic prediction of our proposed friction law 4 (solid thin line without oscillations) and numerical simulations for the center of mass velocity $v_{+}(t)$ vs time $t$. Parameters are $a / b=0.5$ and $\gamma b \sqrt{m / u_{0}}=1$. Solid thick line and broken line are for $k b^{2} / u_{0}$ equal to 0.02 and 10 , respectively. Velocity is expressed in units of $\sqrt{u_{0} / m}$ and time is in units of $b \sqrt{m / u_{0}}$. Initial velocity $v_{0}$ is $5 \sqrt{u_{0} / m}$.

pression (4) and with two numerical simulations made with two different values of the spring constant $k$ separated by a factor of 500. At the time scale of the plot, it is hard to see the difference between the analytical prediction and the simulation with the smaller of the two $k$, while for the larger $k$ the discrepancies between simulation and analytical results are quite evident.

The origin of the periodic dependence of the friction coefficient on the ratio of the equilibrium dimer separation to the substrate wavelength, evident from Fig. 4 and from Eq. (13), should be clear from our analytic argument given at the beginning of the present section. Physically, the importance of this commensurability of dimer and substrate lengths can be understood as follows. When $a$ equals integral multiples of $b$, the two masses constituting the dimer feel exactly the same substrate force when in equilibrium. The overall effect of this in-phase situation is that the internal motion is never excited, and friction disappears. Analytically, this is also clear from the sine term in Eq. (13), which vanishes identically for this case. On the other hand, when $a$ equals semiintegral multiples of $b$, the two masses tend to be precisely out of phase (counterphase), which is the most effective situation to excite the internal motion, and gives rise to maximum friction. Analytically, this is represented by the sine term being equal to 1 .

\section{CONCLUSIONS}

Our general aim in the present paper has been the investigation of some puzzling features of atomic friction. Specifically, we have pursued the dynamics of a model addressing the sliding friction of a dimer. The model is simple: a linear damped oscillator sliding in a sinusoidal periodic potential. Yet, except for the limitation that it is restricted to a single spatial dimension, it has the necessary and sufficient ingredients to be an acceptable model for a real dimer or molecule settled in a controlled microscopic sliding experiment. For example, a molecule sliding along channels of a crystalline well-oriented substrate ${ }^{14}$ might exhibit the described behavior, provided that the temperature is so low that all external 
(electronic) damping may be neglected. Our results (both analytical and numerical) confirm the existence of nonlinear friction and show that it can arise without introducing any explicit damping in the center of mass coordinate. Along with recent studies such as those in Refs. 8, 9, and 13, they open up a field for possible experimental work in order to verify if such a phenomenon can be observed in the microscopic domain.

We mention in passing some related studies in the literature. Tsekov and Ruckenstein ${ }^{15}$ have addressed the problem of diffusion of molecules in solids. They consider a rigid dimer in the external potential of harmonic interacting particles representing the substrate. They find a Langevin equation for the dimer where the effective damping constant is a periodic function of both the position of the dimer and the ratio between the dimer and the lattice length. Our analysis differs from theirs in that our substrate is fixed but our dimer vibrates, the case being precisely the reverse in their system.
If both dynamics were present simultaneously, we expect that both kinds of sliding friction would appear: normal viscous friction derivable from substrate phonons, ${ }^{15}$ and the nonlinear friction because of the internal damped dimer motion that we have analyzed. Such work is under way.

\section{ACKNOWLEDGMENTS}

It is a pleasure to thank Rodrigo Laje and Mukesh Tiwari for numerous discussions. This work was supported in part by a grant made by the Los Alamos National Laboratory to the Consortium of the Americas of the University of New Mexico, and by the National Science Foundation under Grant No. INT-0336343 and DMR-0097204. S.G. acknowledges the hospitality of the Department of Physics and Astronomy of the University of New Mexico, during his stay at the Consortium of the Americas. Work at Los Alamos is supported by the USDOE.
*Electronic address: sgonc@if.ufrgs.br

†Electronic address: kenkre@unm.edu

†Electronic address: arb@lanl.gov

${ }^{1}$ J. Krim, Surf. Sci. 500, 741 (2002).

${ }^{2}$ F. P. Bowden and D. Tabor, The Friction and Lubrication of Solids, 2nd ed. (Clarendon, Oxford, 1986).

${ }^{3}$ J. Krim and A. Widom, Phys. Rev. B 38, 12184 (1988).

${ }^{4}$ M. Cieplak, E. S. Smith, and M. O. Robbins, Science 265, 1209 (1994).

${ }^{5}$ B. N. J. Persson and A. Nitzan, Surf. Sci. 367, 261 (1996).

${ }^{6}$ M. S. Tomassone, J. B. Sokoloff, A. Widom, and J. Krim, Phys. Rev. Lett. 79, 4798 (1997).

${ }^{7}$ A. Liebsch, S. Gonçalves, and M. Kiwi, Phys. Rev. B 60, 5034 (1999).
${ }^{8}$ B. N. J. Persson, Phys. Rev. B 48, 18140 (1993).

${ }^{9}$ C. Fusco and A. Fasolino, Thin Solid Films 428, 34 (2003).

${ }^{10}$ H. Risken, The Fokker-Planck Equation, 2nd ed. (Springer, Berlin, 1989).

${ }^{11}$ B. N. J. Persson, Sliding Friction, 2nd ed. (Springer, Berlin, 2000), and references therein.

${ }^{12}$ R. Tsekov and E. Ruckenstein, J. Chem. Phys. 100, 1450 (1994).

${ }^{13}$ C. Fusco, A. Fasolino, and T. Janssen, Eur. Phys. J. B 31, 95 (2003).

${ }^{14}$ See, e.g., A. H. Romero, A. M. Lacasta, and J. M. Sancho, Phys. Rev. E 69, 051105 (2004), in which an analysis is presented related to such physical situation.

${ }^{15}$ R. Tsekov and E. Ruckenstein, J. Chem. Phys. 100, 3808 (1994). 